\title{
Hybrid Optical and Wireless Sensor Networks
}

\author{
Lianshan Yan, Xiaoyin Li, Zhen Zhang, Jiangtao Liu and Wei Pan \\ Southwest Jiaotong University \\ Chengdu, Sichuan, China
}

\section{Introduction}

Wireless sensor network (WSN) has attracted considerable attentions during the last few years due to characteristics such as feasibility of rapid deployment, self-organization (different from ad hoc networks though) and fault tolerance, as well as rapid development of wireless communications and integrated electronics [1]. Such networks are constructed by randomly but densely scattered tiny sensor nodes (Fig. 1). As sensor nodes are prone to failures and the network topology changes very frequently, different protocols have been proposed to save the overall energy dissipation in WSNs [2-5]. Among them, Low-EnergyAdaptive-Clustering-Hierarchy (LEACH), first proposed by researchers from Massachusetts Institute of Technology [5], is considered to be one of the most effective protocols in terms of energy efficiency [6-7]. Another protocol, called Power-Efficient Gathering in Sensor Information Systems (PEGASIS), is a near optimal chain-based protocol [8].

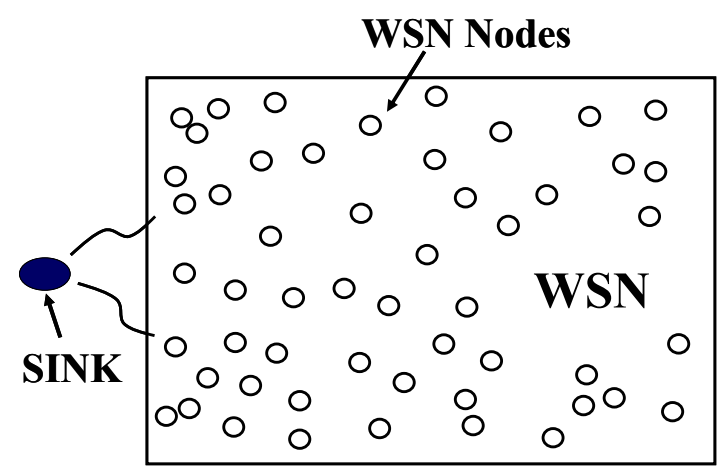

Fig. 1. Illustration of a wireless sensor network (WSN) with randomly scattered nodes (sink node: no energy restriction; WSN nodes: with energy restriction);

On the other hand, distributed fiber sensors (DFS) have been intensively studied or even deployed for analyzing loss, external pressure and temperature or birefringence distribution along the fiber link, ranging from hundreds of meters to tens of kilometers [9-13]. Mechanisms include Rayleigh, Brillouin or Raman scattering or polarization effects, through either time or frequency-domain analysis. Compared with conventional sensors including 
wireless ones, optical fiber sensors have intrinsic advantages such as high sensitivity, the immunity to electromagnetic interference (EMI), superior endurance in harsh environments and much longer lifetime.

Apparently it would be highly desirable to have integrated sensor networks that can take advantages of both WSNs and fiber sensor networks (FSNs). Such hybrid sensor networks can find major applications including monitoring inaccessible terrains (military, highvoltage electricity facilities, etc.), long-term observation of earthquake activity and large area environmental control with tunnels, and so on. So far hybrid sensor networks have been studied as well [14-16], while optical sensors in these networks are generally point-like (e.g. fiber-Bragg-grating based), and such nodes can be regarded as normal WSN nodes after optical to wireless signal conversion.

In this chapter, we first review typical WSN protocols, mainly about LEACH and PEGASIS, then evaluate the performance of LEACH protocols for different topologies, especially the rectangle one. We propose an improved algorithm based on LEACH and PEGASIS for the WSN, finally and most importantly, we propose an O-LEACH protocol for the hybrid sensor network that is composed of a DFS link and two separated WSNs. Most analyses about performance are done in terms of lifetime of the sensor networks.

\section{Overview of WSN protocols}

Wireless sensor networks (WSNs) generally are composed of small or tiny nodes with sensing, computation, and communication capabilities. Various routing, power management, and data dissemination protocols have been specifically designed for WSNs where energy awareness is an essential design issue. Among them, routing protocols might differ depending on the applications and network architectures. In general, routing protocols for the wireless sensor networks can be divided into flat-based, hierarchical-based, and location-based in terms of the underlying network structures [17-19]. As some protocols may be discussed intensively in other chapters of this book, here we give a brief review about major protocols.

(1) Flat-based routing protocol

Sensor nodes in flat-based routing protocols have the same role and collaborate together to perform the sensing task and multi-hop communication. Since the flat routing is based on flooding, it has several demerits, such as large routing overhead and high energy dissipation. Flat-based routing protocol is used in the early stage of WSNs, such as Flooding, Gossiping, SPIN, and Rumor.

(2) Hierarchical-based routing protocol

Hierarchical-based routing protocol is the main trend for WSN's routing protocols. In hierarchical-based routing protocols, the network is divided into several logical groups within a fixed area. The logical groups are called clusters. Sensor nodes collect the information in a cluster and a head node aggregates the information. Each sensor node delivers the sensing data to the head node in the cluster and the head node delivers the aggregated data to the base station which is located outside of the sensor network. Contrary to flat routing protocols, only a head node aggregates the collected information and sends it to the base station. Due to these advantages, sensor nodes can remarkably save their own 
energy. In general, a hierarchical routing technique is regarded as superior to flat routing approaches. The classical Hierarchical-based routing protocols are LEACH, PEGASIS, HPEGASIS, TEEN, and APTEEN. We will discuss the LEACH and PEGASIS protocols in more details later.

(3) Location-based routing protocol

Such protocol is based on the location information of senor nodes in WSNs. It assumes that each node would know its own location and its neighbor sensor nodes' location before sensor nodes sensing and collecting the peripheral information. The distance between neighbouring sensor nodes can be computed based on the incoming signal strength [17-18].

\subsection{LEACH}

Low Energy Adaptive Clustering Hierarchy (LEACH) was first introduced by Heinzelman, et al. in $[5,20]$ with advantages such as energy efficiency, simplicity and load balancing ability. LEACH is a cluster-based protocol, therefore the numbers of cluster heads and cluster members generated by LEACH are important parameters for achieving better performance.

In LEACH protocol, the sensor nodes in the network are divided into a number of clusters, the nodes organize themselves into preferred local clusters, a sensor node is selected randomly as the cluster head $(\mathrm{CH})$ in each cluster and this role is rotated to evenly distribute the energy load among nodes of the network. The $\mathrm{CH}$ nodes compress data arriving from nodes that belong to the respective cluster, and send an aggregated packet to the BS in order to further reduce the amount of information that must be transmitted to the BS, thus reducing energy dissipation and enhancing system lifetime. After a given interval of time, randomized rotation of the role of $\mathrm{CH}$ is conducted to maximize the uniformity of energy dissipation of the network. Sensors elect themselves to be local cluster heads at any time with a certain probability. Generally only $\sim 5 \%$ of nodes need to act as $\mathrm{CHs}$ based on simulation results. $\mathrm{LEACH}$ uses a TDMA/CDMA MAC to reduce intercluster and intracluster collisions. As data collection is centralized and performed periodically, this protocol is most appropriate when there is a need for constant monitoring by the sensor network.

The operation of LEACH is broken up into rounds, where each round begins with a set-up phase followed by a steady-state phase. In order to minimize overhead, the steady-state phase takes longer time compared to the set-up phase. In the setup phase, the clusters are organized and CHs are selected. In the steady state phase, the actual data transfer to the BS takes place. During the setup phase, each node decides whether or not to become a cluster head for the current round. A predetermined fraction of nodes, $\mathrm{p}$, elect themselves as CHs. A sensor node chooses a random number between 0 and 1 . If this random number is less than a threshold value $T(n)$, , the node becomes a cluster head for the current round. The threshold value is calculated based on Eq. (2-1):

$$
T(n)= \begin{cases}\frac{p}{1-p^{*}\left(r \bmod \frac{1}{p}\right)} & \text { if } n \in G \\ 0 & \text { otherwise }\end{cases}
$$


Where $\mathrm{p}$ is the desired percentage of the cluster heads (e.g. $\mathrm{p}=0.05), \mathrm{r}$ is the current round, and $G$ is the set of nodes that have not been cluster heads in the last $1 / p$ rounds. Using this threshold, each node may be a cluster head sometime within $1 / \mathrm{p}$ rounds. All elected $\mathrm{CHs}$ broadcast an advertisement message to the rest of nodes in the network that they are the new CHs. After receiving the advertisement, all non- $\mathrm{CH}$ nodes decide on the cluster to which they want to belong based on the signal strength of the advertisement. The non- $\mathrm{CH}$ nodes then inform the appropriate $\mathrm{CHs}$ to be a member of the cluster. After receiving all the messages from the nodes that would like to be included in the cluster and based on the number of nodes in the cluster, the $\mathrm{CH}$ node creates a TDMA schedule and assigns each node a time slot when it can transmit information. This schedule is broadcast to all the nodes in the cluster. During the steady state phase, the sensor nodes can begin sensing and transmitting data to the $\mathrm{CHs}$. The $\mathrm{CH}$ node must keep its receiver on to receive all the data from the nodes in the cluster. Each cluster communicates using different CDMA codes to reduce interference from nodes belonging to other clusters. After receiving all the data, the $\mathrm{CH}$ aggregates the data before sending it to the BS. This ends a typical round.

Advantages of LEACH include: (i) by using adaptive clusters and rotating cluster heads, LEACH allows the energy requirements of the system to be distributed among all the sensors; (ii) LEACH is able to perform local computation in each cluster to reduce the amount of data that must be transmitted to the base station. On the other hand, there are still some drawbacks about LEACH: (i) LEACH assumes that each node could communicate with the sink and each node has computational power to support different MAC protocols, which limits its application to networks deployed in large regions. (ii) LEACH does not determine how to distribute the $\mathrm{CH}$ s uniformly through the network. Therefore, there is the possibility that the elected $\mathrm{CHs}$ will be concentrated in one part of the network. (iii) LEACH assumes that all nodes begin with the same amount of energy capacity in each election round, assuming that being a $\mathrm{CH}$ consumes approximately the same amount of energy for each node. Hence LEACH is not appropriate for non-uniform energy nodes [17, 20].

\subsection{PEGASIS}

In [8], an enhancement over the LEACH protocol called Power-Efficient Gathering in Sensor Information Systems (PEGASIS) was proposed. The basic idea of the protocol is that nodes only receive from and transmit to the closest neighbours, and they take turns being the leader for communicating with the BS. This reduces the power required to transmit data per round as the power draining is spread uniformly over all nodes. Hence, PEGASIS has two main objectives: (i) to increase the lifetime of each node by using collaborative techniques; (ii) to allow only local coordination between nodes that are close together so that the bandwidth consumed in communication is reduced.

PEGASIS adopts a homogenous topology. In this topology, the BS lies far from sensors with the fixed position. The data is collected and compressed before sent to the next node. Hence the messages maintain ideally a fixed size when they are transmitted between sensors. To locate the closest neighbour node in PEGASIS, each node uses the signal strength to measure the distance to all neighbouring nodes and then adjusts the signal strength so that only one node can be heard. The chain in PEGASIS consists of those nodes that are closest to each other and form a path to the BS. The following describes the protocol briefly: 
1) The chain starts with the furthest node from the BS to make sure that nodes father from the BS have close neighbours. Based on the greedy algorithm, the neighbour node joins into the chain with its distance increases gradually. When a node dies, the chain is reconstructed in the same manner to bypass the dead node.

2) To gather data in each round, a token is generated by the BS to set the aggregating direction after the token sent from the BS to an end node. Each node receives data from one neighbour, fuses with its own data, and transmits to the other neighbour on the chain.

3) Only one node transmits data to the BS in certain rounds, the leader is the node whose number is ( $\mathrm{i} \bmod \mathrm{N})$ where $\mathrm{N}$ represents the number of the nodes in round $\mathrm{i}$.

PEGASIS is better than LEACH in terms of energy saving due to following facts: (i) During the data localization, the distances that most of the nodes transmit information are much shorter compared to that in LEACH. (ii) The amount of data for the leader to receive is much less than LEACH. (iii) only one node transmits to the BS in each round.

Though PEGASIS has obvious advantages, it has some shortcomings. Firstly, though most sensors are joined on a chain to form a basically homogenous structure, a sensor with too much branches may perform many times of data receiving in a certain round thereby resulting in unbalanced energy problem. Secondly, all the nodes must keep active before the token arriving. This means there will be a large percentage of active nodes with nothing to do from the beginning, meaning a waste of energy and time. Thirdly, once a sensor on the chain was captured the whole net may be under the control by the attackers. The weak security could be a great threat $[17,20]$.

LEACH that is a cluster-based protocol and PEGASIS that is a chain-based protocol are the most classical Hierarchical-based routing protocols. They both have attracted intensive attention, and lots of routing protocols are based on these two. Next we will investigate some issues in details.

\section{WSN Topologies}

\subsection{Shapes of different topologies}

According to the shape of WSN monitoring area, application requirements and monitoring of different targets, different topologies should be chosen for deploying the WSN: circular topology is preferred for applications such as harbour, stadium etc. [21]; square topology is suitable for irrigation in agriculture, nature reserve area etc.; rectangular topology can be chosen for highway, railway, mine and other areas [22].

Here we study the life time of WSN in round, square, rectangular shapes of topology, and the three topologies are shown in Figs. $3.1(\mathrm{a}-\mathrm{c})$. In Fig. 3.1(a), the circular area is 10,000 $\mathrm{m}^{2}$ (same as square, rectangular areas) with the radius $\mathrm{R}=56.419 \mathrm{~m}$ and the base station is located at the center of the circle, i.e. $(0,0)$. In Fig. 3.1(b), the size of the square area is $100 \times 100 \mathrm{~m}^{2}$ with the base station located on $(0,50)$ or $(50,175)$. In Fig.3.1(c), the size of the rectangular area is $50 * 200 \mathrm{~m}$ with the base station located on $(0,25)$ or $(100,150)$. 


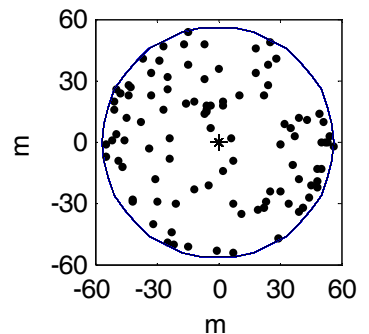

(a) Circular topology

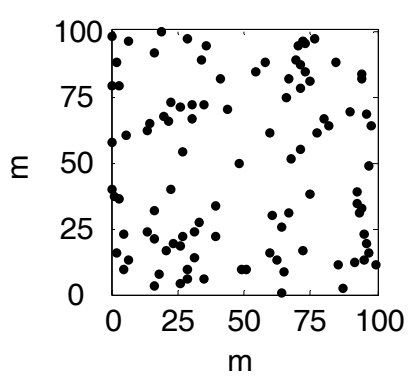

(b) Square topology

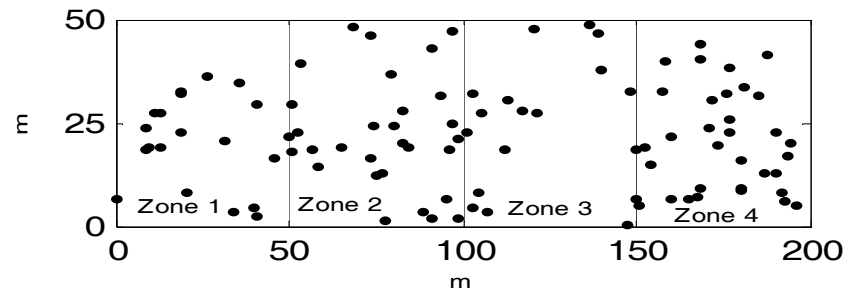

(c) Rectangle topology

Fig 3.1 Three different types of WSN's topology:

Sink $(\mathrm{a}):(0,0) ;(b):(0,50)$ or $(50,175) ;(c):(0,25)$ or $(100,150)$

The probability of cluster head node in the LEACH protocol has a certain impact on the WSN's lifetime. In our analysis, we divides the rectangle area into four smaller square areas, with the communication distance of nodes keeping short $\left(\mathrm{d}_{\mathrm{B}}<\mathrm{d}_{0}\right.$, where $\mathrm{d}_{\mathrm{B}}$ is the broadcasting distance of the cluster head). From the first-order radio model, we can get the optimal cluster head probability formula as follows:

$$
k_{o p t}=\frac{\sqrt{N}}{\sqrt{2 \pi}} \cdot \frac{M}{d_{t o B S}}
$$

Where $\mathrm{N}$ is the number of sensor nodes. In the rectangular region 100 nodes are scattered randomly, and the region is divided into four regions. In order to verify the difference of the number of nodes distributed in different regions, we simulate 100 independent iterations of the nodes' number in each region, and get the averages in the four regions as zone1(25.32)、 zone2(24.83)、zone3(24.87) and zone4(24.98). It can be seen that the number of nodes in each region are around 25 , there're almost no difference in the average nodes' numbers for the four regions, so in the text, the nodes are uniform distributed in the four regions, i.e. $\mathrm{N}=25 . \mathrm{M}$ is the side length of each small square region, here $\mathrm{M}=50 . \mathrm{d}_{\mathrm{toBs}}$ is the distance between the sink and the node. As the distance of a node to the base station is different, we can change the percentage of cluster heads in different regions to reach the optimal value so that the lifetime of the whole WSN can be prolonged.

For the topology with a rectangular shape, we propose an improved LEACH algorithm. The main idea is described as follows: 
(1) Divide the rectangular area into several small square areas with the same size;

(2) Elect the cluster heads separately in each region, and the optimal probabilities of the cluster heads for each region can be obtained from Eq. (3-1), i.e. the values are $\mathrm{p}_{1}=0.02, \mathrm{p}_{2}=0.03, \mathrm{p}_{3}=0.03, \mathrm{p}_{4}=0.02$;

(3) After the cluster heads in each region are selected, the rest of the protocol is similar to LEACH.

The improved algorithm elects cluster heads in each region according to its probability of the cluster heads. In this way, it can make sure that there are clusters in every region and ensure the clusters distributed more uniformly in every region, which reduces the energy dissipation and improves the lifetime of the network.

\subsection{Simulation results}

We simulate the three shapes of topology that use (improved) LEACH as the routing protocol. Parameters used in simulation are listed in table 3.1. There are 100 sensor nodes randomly scattered with fixed position in each shape. We measure the round number when the first node died, $20 \%$ of nodes died and $50 \%$ of nodes died respectively as the criterion to estimate the lifetime of WSN.

Table 3.1 Parameters used in simulation

\begin{tabular}{lc}
\hline Parameter & Value \\
\hline Number of nodes & 100 \\
Initial energy (J) & 0.5 \\
Data packet length (bit) & 4000 \\
Control packet length(bit) & 200 \\
Energy dissipation of one Tx (nJ) & 50 \\
Energy dissipation of one Rx (nJ) & 50 \\
Energy aggregation energy (nJ) & 5 \\
Energy loss-free space (pJ/bit/m $\left./ \mathrm{m}^{2}\right)$ & 10 \\
Energy loss-multipass fading $\left(\mathrm{pJ} / \mathrm{bit} / \mathrm{m}^{4}\right)$ & 0.0013 \\
\hline
\end{tabular}

Table 3.2 shows the round numbers (the lifetime) of WSN for different BS locations and different percentage of dead nodes in circle, square and rectangular shapes of topology. As the sensor nodes distributed randomly in WSN that may statistically vary, we simulate every case for 100 iterations to get more accurate results. The percentage of the cluster heads is set to $5 \%$ in three shapes of topology. It can be seen from Table 3.2 that the longest lifetime of WSN is the circle shape of topology. The BS located in the center of the circle that is symmetric, so that the energy dissipation of nodes are more even and the lifetime of the network is prolonged. For the rectangle shape, the lifetime is different as the position of BS changes. Simulation results indicate that the lifetime for the BS in $(0,50)$ is longer that in $(50,175)$. As the BS in $(0,50)$ is nearer to the sensor area, the energy for transmitting data to the BS is reduced. 


\begin{tabular}{cccccc}
\hline Topology & Circle & \multicolumn{2}{c}{ Square } & \multicolumn{2}{c}{ Rectangle } \\
\hline Sink & $(0,0)$ & $(0,50)$ & $(50,175)$ & $(0,25)$ & $(100,150)$ \\
$1 \%$ & 757 & 740 & 639 & 470 & 555 \\
$20 \%$ & 854 & 846 & 715 & 644 & 652 \\
$50 \%$ & 923 & 919 & 788 & 785 & 717 \\
\hline
\end{tabular}

Table 3.2 Lifetime comparisons of different shapes of topology

It can be seen form Table 3.2 that the lifetimes of the rectangular are poor for the BS both near and far away from the sensor area using conventional LEACH protocol. Then we use the improved LEACH algorithm that divides the rectangle region into four equal square regions. The cluster heads are elected separately in each region to make sure the cluster heads distribute uniformly in four regions.

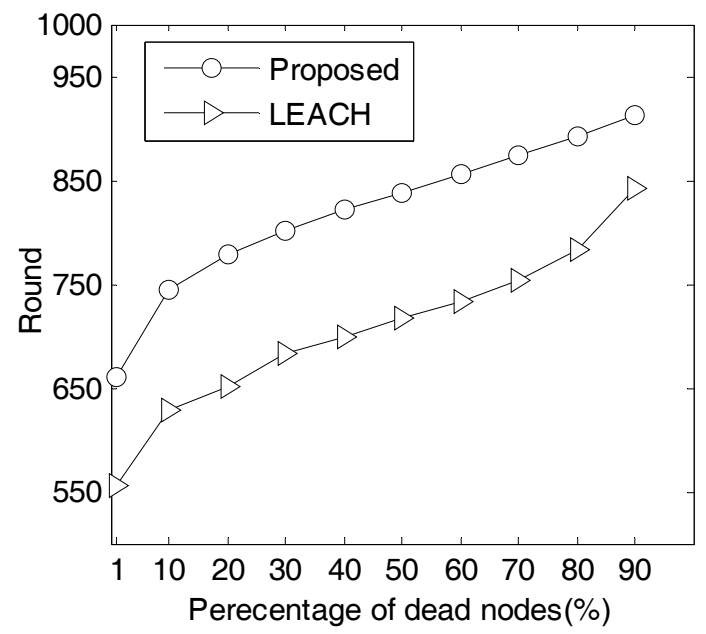

Fig 3.2 The relationship between percentage of dead nodes and number of rounds

Fig. 3.2 shows the improvement using the modified LEACH algorithm in terms of the lifetime of the network. It can be seen from the figure that the surviving round number of improved algorithm is increased under the same percentage of dead nodes, corresponding to the lifetime improvement of $19 \%$ (for the case of the first died node) compared with the conventional LEACH algorithm. This is due to the fact that the improved algorithm reduces the energy dissipation by dividing the rectangle region into sub-regions.

From the above analysis, when sensor nodes within a monitoring area can be manually deployed, one can select the circle topology of the WSN to maximize the lifetime. When the topology area is square and the base station location is variable, one can make the base station close to the WSN area to save energy and extend the network lifetime. When the monitoring area is a rectangular one, one can use the idea of partition to extend the lifetime. 


\section{LEACH \& PEGASIS}

\subsection{Introduction of LEACH \& PEGASIS algorithm}

Brief introductions about LEACH and PEGASIS have been given in section 2. As pointed, there are three shortcomings for the LEACH protocol:

(1) The number of cluster heads is uncertain. If the number of cluster heads is large, cluster heads that need communicate directly with the base station will consume more energy. If the number of cluster heads is small, common nodes that need communicate with the remote cluster heads will consume more energy.

(2) When cluster heads are selected, the remaining energy of cluster heads is not considered. After a node is elected as the cluster head, maybe the remaining energy is not enough for the next round of communication, therefore may lead to failure of the entire cluster, and member nodes of the cluster will lose data. A blind spot will appear within the monitoring area.

(3) Many cluster heads communicate directly with the base station. Especially the cluster heads are far away from the base station. Transmission of data consumes a lot of energy. As the cluster head dies prematurely, the total energy of network consumes excessively.

To overcome the shortcomings of LEACH, we take the advantages of PEGASIS to construct a chain using greedy algorithm which only uses a node as the cluster head to communicate with the base station. Studies show that the approach of data fusion and multi-hop based on cluster can save the energy of nodes even better [23]. Here we propose an improved routing protocol called LEACH-P.

(1) The optimal number of cluster head is defined by Eq. (4-1).

$$
m=\frac{\sqrt{N}}{\sqrt{2 \pi}} \sqrt{\frac{\varepsilon_{f s}}{\varepsilon_{m p}}} \frac{M}{d_{t o B S}^{2}}
$$

Where $\mathrm{m}$ is the optimal number of cluster heads; $\mathrm{N}$ is the number of nodes; $\varepsilon_{\mathrm{fs}}$ is signal amplification factor in free space; $\varepsilon_{\mathrm{amp}}$ is signal amplification factor of the multipath fading channel; $M$ is the side length; $d_{\text {toBs }}$ is the distance between the cluster head and the base station.

(2) Cluster heads are decided by Eq. (2-1) in the LEACH algorithm without taking into account the residual energy of nodes. The new algorithm detects the residual energy of the cluster head to meet the required energy threshold $\mathrm{E}(\mathrm{r})$, i.e. the minimum energy to complete one round communication, which is sum of the energy for broadcasting information, receiving data packets and confirming messages from cluster members to, as well as communicating with its neighboring cluster heads.

In our simulation (parameters and definitions of acronyms are listed in Table. 4.1. We assume that the coverage of broadcasting is half of the diagonal of the field, which is less than $\mathrm{d}_{0}$. Energy consumed through broadcasting is:

$$
E T X * c P L+\varepsilon_{f s} * c P L * D B^{2}
$$


The energy that each cluster head needs to receive confirmation and data packets from its cluster members is:

$$
19 *\left(\left(E R X+E_{D A}\right) * P L+E R X * c P L\right.
$$

In each cluster, the average number of nodes that the cluster head needs to receive the information is 19 .

The energy that the cluster head needs to send information to its neighboring cluster head is:

$$
\left(E T X+E_{D A}\right) * P L+\varepsilon_{f s} * P L * D B^{2}
$$

The distance between cluster heads is random, and we assume it as the broadcasting distance between nodes.

From above, we can obtain that the energy threshold value is $\sim 0.0048 \mathrm{~J}$.

(3) The new algorithm (LEACH-P) randomly selects five cluster heads linked as a chain. The one with maximum residual energy is chosen to transfer information to the sink. Other cluster heads reduce energy dissipation through data fusion. LEACH-P uses the wireless communication model described in [24]. The energy dissipation that transmits K-bit data to the receiver over a certain distance $\mathrm{d}$ is:

$$
E_{t}(k, d)= \begin{cases}k E_{\text {elec }}+k \varepsilon_{f s} d^{2} & d<d_{0} \\ k E_{\text {elec }}+k \varepsilon_{m p} d^{4} & d \geq d_{0}\end{cases}
$$

Where $\mathrm{E}_{\text {elec }}$ is the energy dissipation of the transmitter; $\varepsilon_{\mathrm{fs}}$ and $\varepsilon_{\mathrm{mp}}$ are the energy dissipations of the power amplifiers; $\mathrm{d}_{0}$ is the constant. The energy that a node needs to receive K-bit data is:

$$
E_{r}(k)=k E_{\text {elec }}
$$

The energy to fuse a number $(\mathrm{L})$ of K-bit packets is:

$$
E_{f}(L, k)=L k E_{D A}
$$

Where $\mathrm{E}_{\mathrm{DA}}$ is the energy dissipation of fusing 1-bit data.

The advantage of the PEGASIS link performance has been described and proved in [25]. The topology of the improved algorithm is shown in Fig 4.1. In the figure, the nodes within a cluster have the same symbol. There are five cluster heads with 5 symbols, one of them is elected as the leading cluster head that is responsible for the information exchange with the base station. 


\begin{tabular}{lc}
\hline Parameter & Value \\
\hline Sink location & $(50,175)$ \\
Sensing region & $100 \times 100$ \\
The number of nodes(N) & 100 \\
Initial energy(E) $(\mathrm{J})$ & 0.5 \\
Data packet length(PL) (bit) & 4000 \\
Control packet length(cPL) (bit) & 200 \\
Energy dissipation of one (ETX) (nJ) & 50 \\
Energy dissipation of one (ERX) (nJ) & 50 \\
Energy aggregation energy(EDA) (nJ) & 5 \\
Energy loss-free space(Efs) (pJ/bit/m $\left.\mathrm{m}^{2}\right)$ & 10 \\
Energy loss-multipass fading (Emp) (pJ/bit/m $\left.\mathrm{m}^{4}\right)$ & 0.0013 \\
\hline
\end{tabular}

Table 4.1 Parameters used in simulation

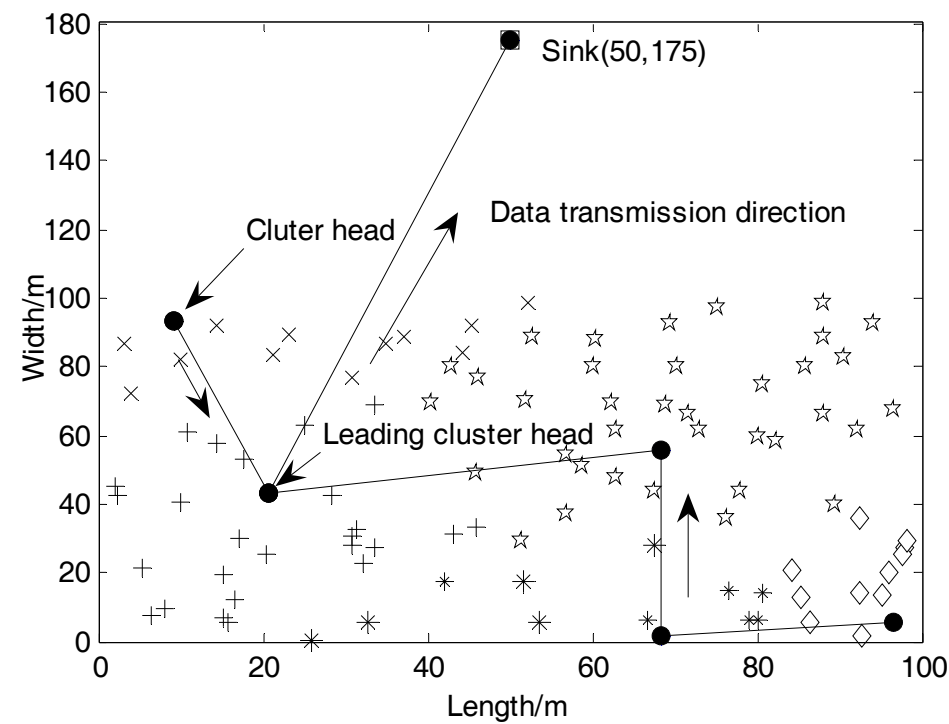

Fig. 4.1 Topology of the improved algorithm

The new algorithm not only solves the problem in the LEACH protocol that multiple cluster heads communicate directly with the base station to introduce more energy dissipation, but also overcomes the shortcoming of the long delay in the PEGASIS protocol. The flowchart of the algorithm is shown in Fig 4.2. In each round of communication, the improved algorithm is still divided into two stages : the setup stage and the stable stage.

In the setup stage, the election of cluster head depends on whether there is any dead node and the residual energy of cluster heads must be greater than $\mathrm{E}(\mathrm{r})$. After the election of cluster heads, the cluster heads broadcast information (advertisement message, ADV). Noncluster head node chooses the cluster according to the signal strength after receiving the 
information, and sends a request that includes the cluster ID, its own ID, as well as its remaining energy state to join the cluster. Cluster heads are connected into a chain after the establishment of clusters, and the cluster head with maximum residual energy is assigned as the leading cluster head to communicate with the BS directly.

In the stable stage, common nodes of the cluster send information to the cluster head according to the TDMA time slot table. The cluster head receives data and integrates data into a packet. The packets are transmitted along the chain to the leading cluster head according to the Token. The leading cluster head receives and fuses data packets, then sends to the base station.

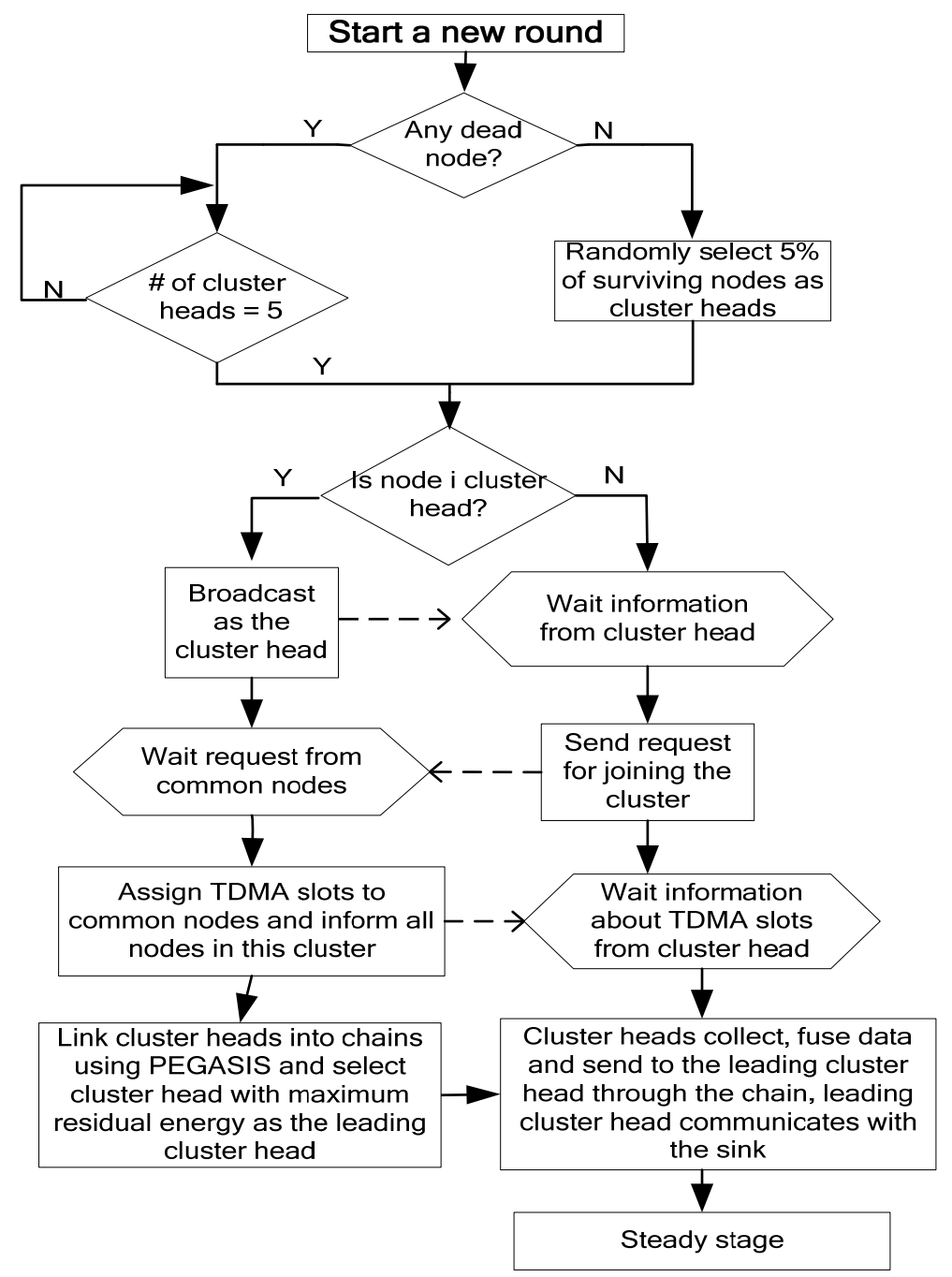

Fig. 4.2 Flow chart of proposed LEACH-P algorithm 


\subsection{Simulation results and analysis}

The performance of improved algorithm (LEACH-P) is evaluated in terms of the lifetime of the network and data transmission delay. 100 sensor nodes are randomly distributed in the sensing area of $100 \times 100 \mathrm{~m}^{2}$. Most simulation parameters are the same as those of the LEACH algorithm, with specific parameters listed in Table 4.1.

The lifetime in terms of rounds corresponding to $1 \%, 20 \%, 50 \%$ of nodes died are simulated for both the LEACH and improved algorithm. The results are compared in Table 4.2. The lifetime of the new algorithm improves by $17 \%$ for the case with $1 \%$ dead nodes. The numbers of rounds when $20 \%$ and $50 \%$ of nodes died are also improved compared with the LEACH protocol. Similar to previous simulation, we take 100 iterations for each case to reduce the statistical fluctuation.

\begin{tabular}{ccc}
\hline Percentage of dead nodes & LEACH & LEACH-P \\
\hline $1 \%$ & 639 & 751 \\
$20 \%$ & 715 & 848 \\
$50 \%$ & 788 & 919 \\
$100 \%$ & 1296 & 1276 \\
\hline
\end{tabular}

Table 4.2 Lifetime comparisons of different algorithms

Fig 4.3 also shows the lifetime comparison for the LEACH and improved algorithm. It can be seen that the round number corresponding to the first dead node and all dead nodes is $\sim 639$ and 1240 for the LEACH protocol, respectively. These two numbers are improved to $\sim 751$ and 1300 using the new algorithm. The Lower part of Fig 4.3 shows the variation of the cluster head number in the new algorithm with a fixed number as 5 until $\sim 800$ rounds (dead nodes appear). Compared with $\mathrm{LEACH}$, the proposed algorithm can prolong the network lifetime and balance the energy dissipation of network nodes as well.

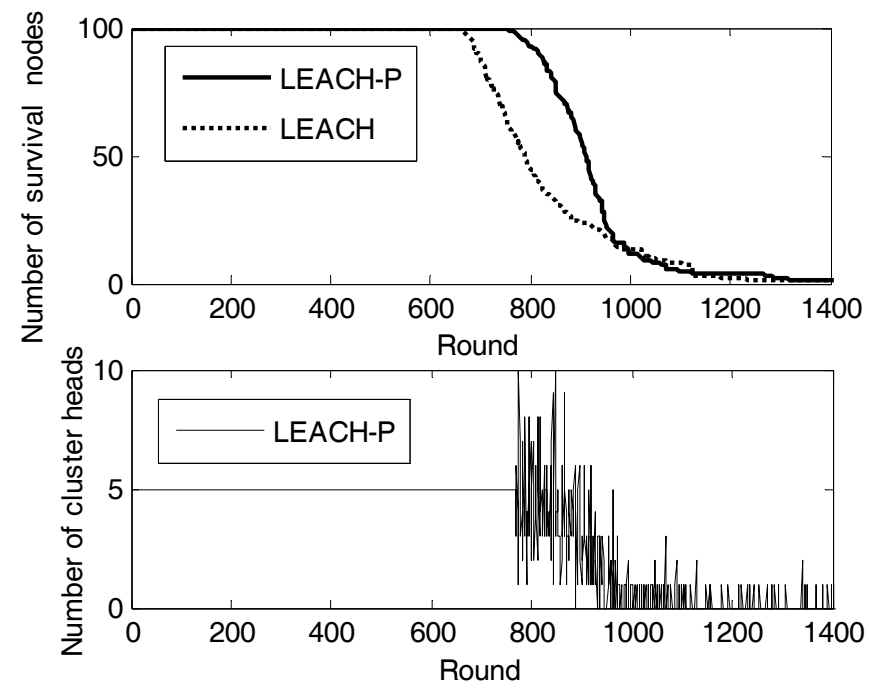

Fig. 4.3 Relationship between rounds and number of survival nodes 
WSNs are generally deployed in harsh environments, where the base station is far away from the sensor field. The location of the base station has a great effect on the lifetime of the sensor network. When the distance between the base station and the monitored region is too large, some cluster heads will lead to excessive energy dissipation in the LEACH, shortening the lifetime of the network. On the other hand, the leading cluster head transmits data to the base station gathered from other cluster heads using multi-hop in the new algorithm. Therefore, the increasing distance between the base station and the monitored regions has less effect on the network lifetime. In Fig. 4.4, the network lifetime of LEACH and proposed algorithm are calculated as we vary the distance between the BS and sensor field. It can be seen from Fig. 4.4 that:

(1) When the base station location changes from 100 to 250, the lifetime of WSN for the LEACH-P algorithm keeps almost constant ( 760). When using the LEACH algorithm, the lifetime is reduced from 737 to 328 .

(2) When the base station location changes from 250 to 400, the lifetime of LEACH-P algorithm and LEACH algorithm are both reduced, but LEACH-P algorithm is still better than the conventional LEACH.

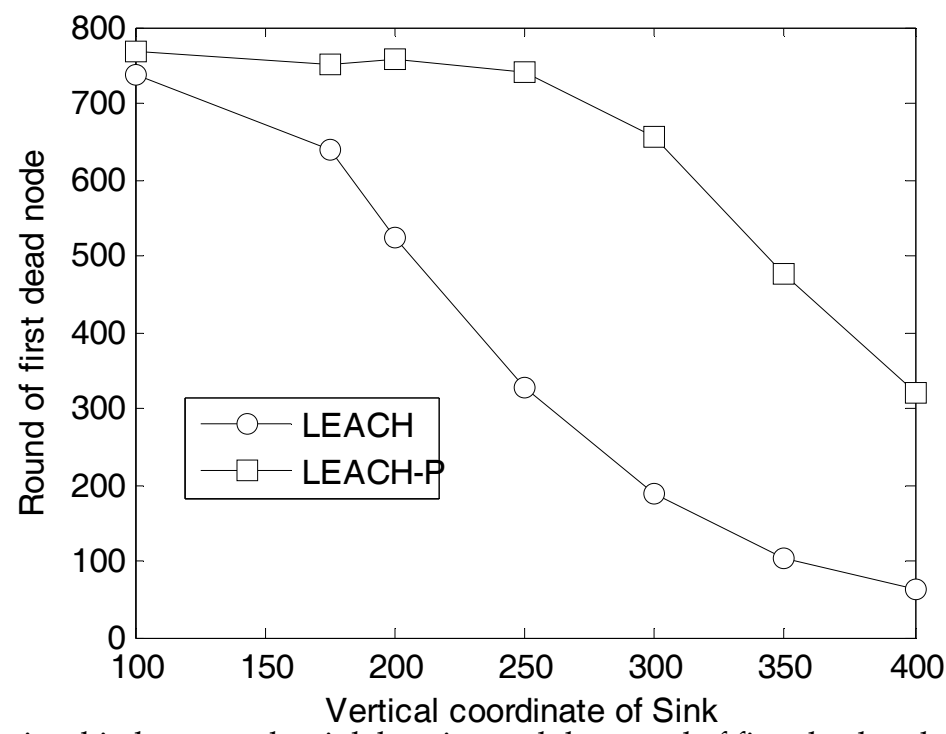

Fig. 4.4 Relationship between the sink location and the round of first dead node

Furthermore, Fig. 4.5 shows the numbers of live nodes using LEACH-P and LEACH protocols with the position of sink fixed at $(50,300)$. Now the number of the first dead node for LEACH is only 187, but it is improved to 658 for the LEACH-P algorithm. Compared with LEACH, LEACH-P can prolong the network lifetime by $351 \%$. Figs. 4.4 and 4.5 further indicate that LEACH-P is superior to LEACH with more evenly energy distribution among nodes even the sink location is far away from the sensor field. 


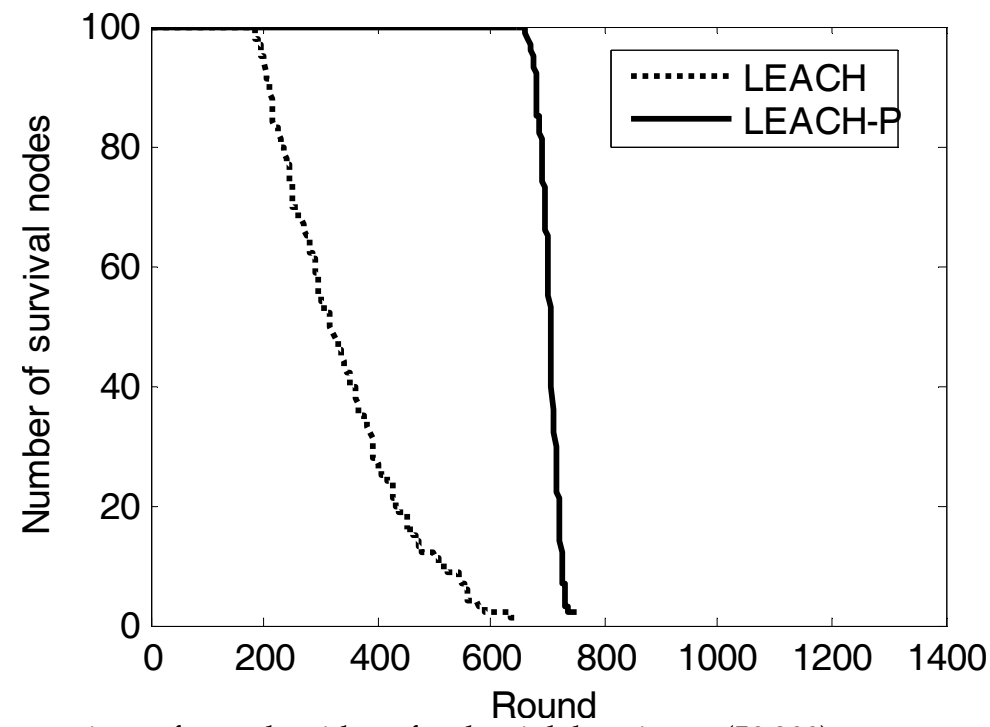

Fig. 4.5 Comparison of two algorithms for the sink location at $(50,300)$

Another significant advantage of LEACH-P is that it not only overcomes the issue of long delay of PEGASIS, but also inherits the idea of greedy into a chain. In order to verify the improvement of the transmission delay of the WSN, we calculate and compare the maximum distance that the data should be transmitted for every round in LEACH, PEGASIS and LEACH-P.

(1) When data is transmitted according to LEACH, all common nodes of the cluster send data to the cluster head in accordance with the TDMA time slots. The cluster head will fuse the data and send it to the sink. The longest distance of transmitting data in each round corresponds to the maximum distance of the sum of both common nodes to the cluster head and the cluster head to the sink.

(2) When data is transmitted using the token mechanism in PEGASIS, there is only one token which goes through the whole chain. The longest distance of transmitting data in each round corresponds to the sum of the distances of both the length of the entire chain and the leading cluster head to the sink.

(3) When data is transmitted using the TDMA and token mechanism in LEACH-P, in each cluster, the cluster head allocates TDMA time slot to common nodes. Common nodes send the data to the cluster head. Between cluster heads, the cluster heads are linked into chains and then transfer the data to the leading cluster head according to the token mechanism. The leading cluster head fuses the data and sends it to the sink. The longest distance of transmitting data in each round corresponds to the sum of the maximum distance of common nodes to cluster heads in two ends of the chain, the length of the chain and the distance between the leading cluster head and the sink.

Again, we simulate all cases with 100 iterations to get the statistical average value. The performance (network delay) is evaluated in terms of the average distance before the first dead node appearing in wireless sensor network. 


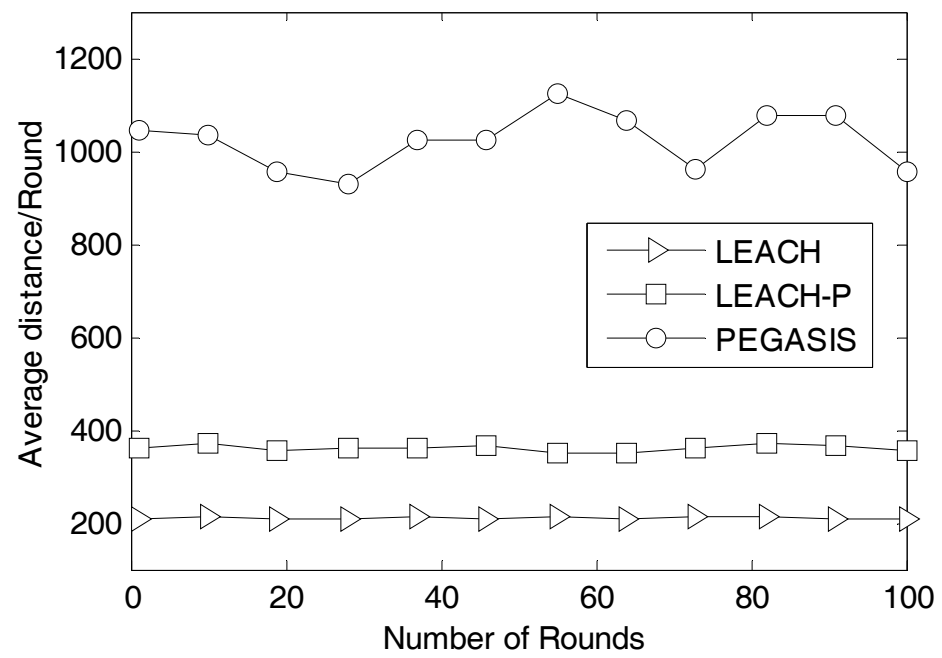

Fig. 4.6 Comparison of transmission delay in terms of maximum distance for three protocols

Fig. 4.6 illustrates the simulation results. In PEGASIS algorithm, the distribution of nodes and the chain length are quite different from round to round, so the average value is still fluctuating greatly. The average longest distances of transmitting data in each round for PEGASIS, LEACH and LEACH-P are $1026.7 \mathrm{~m}, 209.9 \mathrm{~m}$ and $357.33 \mathrm{~m}$, respectively. The realtime of LEACH-P is slightly lower than LEACH. However, compared with PEGASIS, LEACH-P increases by a dramatic value, i.e. $\sim 290 \%$.

Therefore, we can conclude for this section that LEACH-P combines the advantages of both LEACH and PEGASIS. It can not only reduce the energy dissipation of cluster heads compared to the LEACH algorithm in large-scale sensor networks, but also overcome the issue of poor real-time in the PEGASIS algorithm.

\section{O-LEACH}

Above sections are mainly about randomly scattered WSN nodes with different topologies. However, in some particular areas that are difficult to place wireless sensor nodes, we can lay distributed fiber sensor (DFS) along. DFS can achieve measurements such as temperature, strain /stress and so on, which associates with wireless sensor nodes to construct a new hybrid optical wireless sensor network. Incorporating distributed optical fiber sensor in rectangular topological region makes the WSN more suitable to work in harsh and large-scale regions. Meanwhile, the reliability and security of system and data are further protected.

\subsection{O-LEACH algorithm description}

We investigate an infrastructure of hybrid sensor network which is composed of a DFS link and two separated WSNs, as shown in Fig 5.1. The DFS link is located at the center of the whole sensor field and can cover a certain area. The two WSN fields are filled with 
randomly scattered nodes as usual. These nodes can communicate with each others. Unlike simple WSNs, since the DFS has to be powered on for data processing, we use one end of the DFS as the sink or the base station for all WSN nodes. We specially propose a new energy efficient communication protocol, optical LEACH (O-LEACH), based on the WSN LEACH protocol.

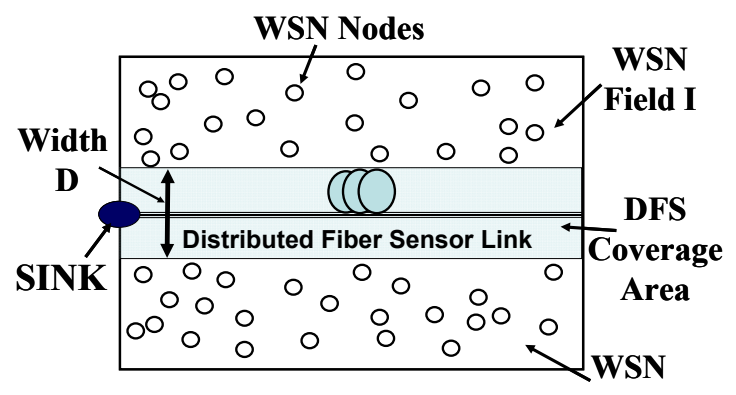

Field II

Fig. 5.1 Sensor field consisting of a distributed fiber sensor (DFS) link and two WSN fields (I and II), the sink node is located at one end of the DFS and the width of the DFS coverage area is D.

As a more general topology, Fig. 5.2 shows such hybrid sensor networks that have potential to cover much more broad areas under certain guidelines: (1) cascade of multiple rectangular regions in which the base station location of sensor node is $(100,150),(2)$ the DFS is located in the monitor area with massive volume of data, harsh environment and poor security (located in the middle of the rectangular region for this paper) to link the rectangular region and the location of fiber's base station is $(0,25)$. The DFS can cover a certain area, for example $10 \mathrm{~m}$ (vertical axis), to monitor the pressure or temperature information within the coverage area, and give the data back to the fiber base station.
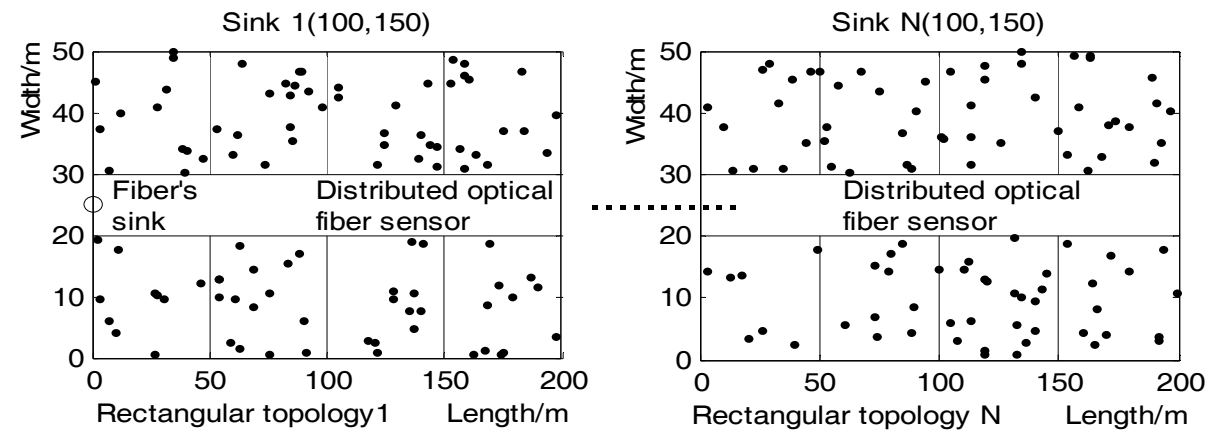

Fig. 5.2 The topology incorporating distributed optical fiber sensors

As nodes of two WSNs are power limited, the protocol is mainly dealing with these nodes. The flowchart of the O-LEACH protocol is shown in Fig. 5.3. As the operation of the standard LEACH protocol is separated into the setup phase and the steady phase, we also 
separate the O-LEACH operation into two phases. The steady phase is as same as the LEACH one. During the setup phase, there are two major differences between O-LEACH and LEACH: (1) nodes of WSNs cannot be deployed in the DFS coverage area; (2) the cluster head and the node should be within the same WSN field if two WSNs cannot communicate with each other (i.e. checking if Position Cluster_head $_{2}=$ Position $_{\text {Node_I }_{-}}$in the flowchart). For most applications, it would be better to assume that two WSN fields are isolated due to the following reasons: (1) save information transfer energy, as transferring data over the DFS terrain would waste more energy; (2) wireless communication over the DFS area is not allowed for some applications. However, we simulate the case that nodes inside different WSN fields can communicate with each other as well for reference.

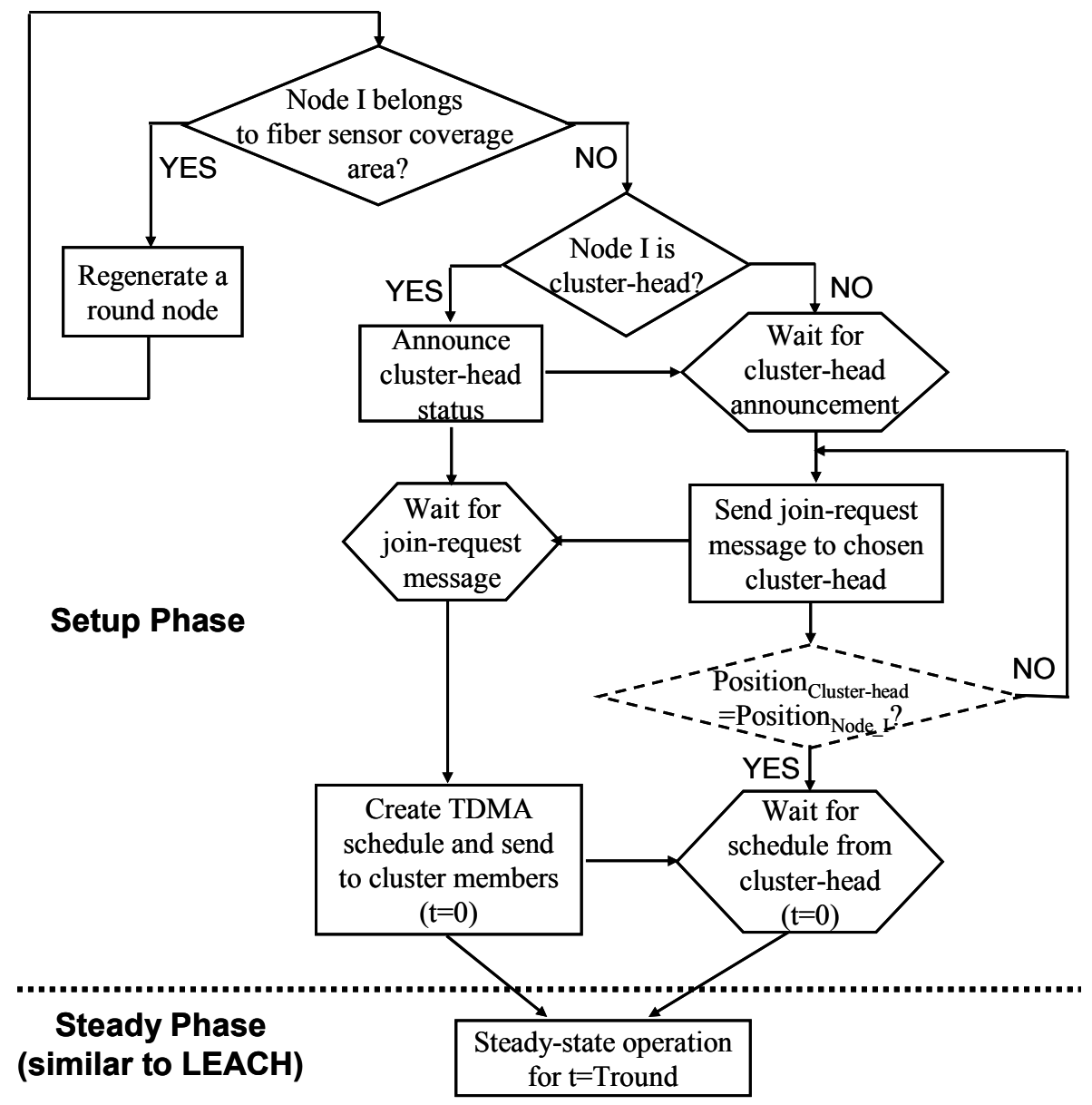

Fig. 5.3 Flowchart of the O-LEACH protocol 


\subsection{Simulation results}

Based on the proposed O-LEACH protocol, we simulate the network performance in terms of the node lifetime. In our simulation model: (1) most of parameters (e.g. probability of a node to become a cluster head, data packet length, control packet length, etc.) are as the same as other LEACH-based simulation models (listed in Table 4.1); (2) the position of the sink in the LEACH model can be put in different places, while in our LEACH and OLEACH ones, we put the sink of all the cases at the same position, i.e. in the middle of one edge of the sensor field; (3) as the network energy dissipation is a totally statistical behavior due to the random distribution of WSN nodes, we simulate every case for 1000 independent iterations (over days).

First we compare the network performance in terms of lifetime for the simple topology shown in Fig. 5.1. Three cases are simulated: original LEACH without DFS; O-LEACH with varying width of DFS coverage area (D) that two WSNs can either communicate with each other or not. As people use different parameters to evaluate the lifetime, i.e. the round number corresponding to the appearance of the first dead node, half of the dead nodes or the last survival node (refered as "first-dead", "half-dead" and "fully-dead" in the following part of the paper), we obtain all the three parameters and find that the network improvement may end up with quite different conclusions through these parameters.

For the LEACH case, we obtain that the average round number corresponding to "firstdead", "half-dead" and "fully-dead" are 731, 915, and 1741, respectively. Figs 5.4 (a) and (b) show the average lifetime evaluated by three "-dead" parameters for situations that the two WSNs can communicate with each other or not as we vary the value of D (from 5 to 50 ). We can see that: (1) the network performance in terms of lifetime keeps almost constant regardless the width of DFS coverage. (2) In the case that two WSNs cannot communicate with each other, nodes can save energy on broadcasting over smaller area (i.e. shorter distance), therefore, the average lifetime corresponding to either "first-dead" or "half-dead" is improved $\sim 20 \%$ compared to the case that two WSNs are connected, while the last node's lifetime ("fully-dead") is more than doubled. (3) Compare to the conventional LEACH protocol, if two WSNs can communicate with each other, the improvement of O-LEACH is very limited $(\sim 2 \%$ and $\sim 16 \%$ in terms of "first-dead" and "fully-dead", respectively). Therefore, it is expected and mostly required for such hybrid sensor networks to employ OLEACH with two isolated WSNs. Furthermore, typical lifetime evolutions are compared as well in Fig. 5.4 (c) where D equals 20. These curves are specially chosen from thousands of simulated iterations with performance close to average ones. Results of $\mathrm{LEACH}$ protocol are also included. 


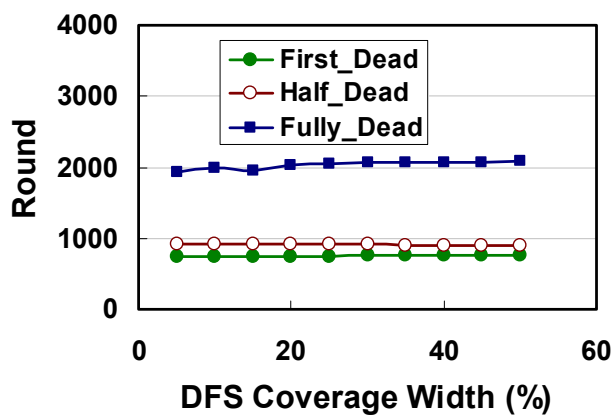

(a)

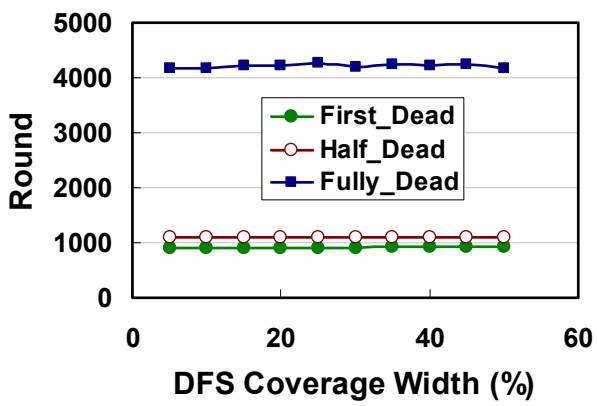

(b)

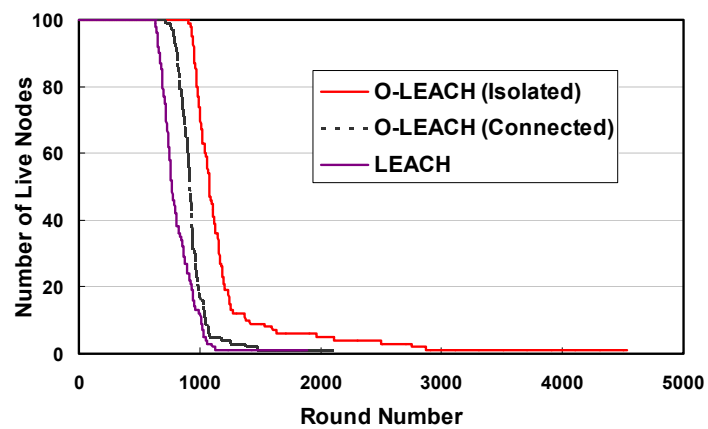

(c)

Fig. 5.4 Simulation results of network performance in terms of lifetime (round number) using O-LEACH protocol: (a) two WSNs can communicate with each other; (b) two WSNs are isolated; (c) typical lifetime evolution curves

A legitimate question for above network model is how close to the reality of the hybrid sensor network in terms of the coverage of DFS and WSN. As pointed in the introduction section, the distance of typical DFS link can vary from hundreds of meters to tens of kilometers, while the coverage diameter of WSN node is tens of meters. Therefore, it would be interesting to look into the case that the length of DFS link increases and the number of WSN nodes increases proportionally (to keep the approximate density).

To keep straightforward but simple, we fix the width of the whole sensor field to 100 and the coverage percentage of DFS to $20 \%$ (i.e. D equals to 20). Also we only consider the case that two WSNs are isolated. Fig. 5.5 illustrates the trend of normalized lifetime performance with increasing link length of DFS. The normalization is done using the ratio of the "-dead" parameter to the total node number. It is obvious that 100 is the optimum number for WSN nodes with parameters listed in Table I. As the length of the DFS link increases, the lifetime reduces dramatically, especially the "first-dead" parameter. More wireless sinks are required for longer DFS links, and the performance evaluation of various optical wireless sensor network topologies are of great interests for further investigation. On the other hand, our protocol and the simulation model can be adapted into networks with different parameters so that we can find the optimized network design. 


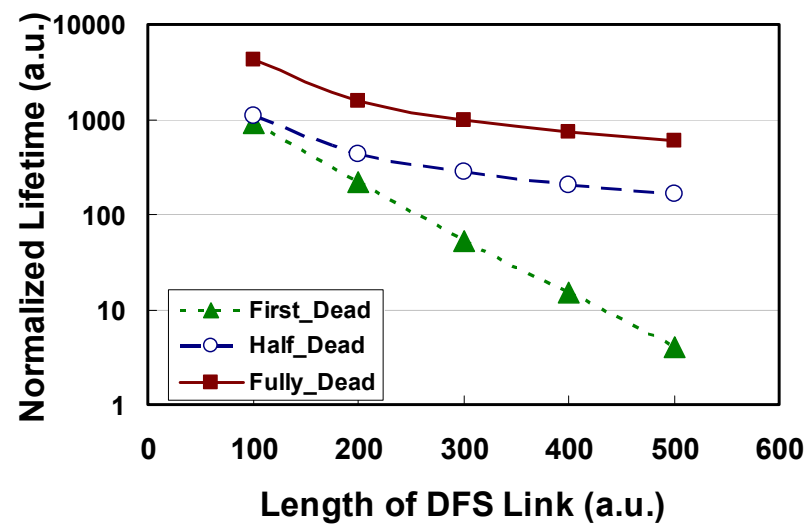

Fig. 5.5 Normalized lifetime as we increase the length of DFS link (number of WSN nodes are increased proportionally, the widths of the DFS coverage area and the whole sensor filed are fixed)

For the general topology shown in Fig.5.2, we simulate a 50*200m rectangular WSN region that is divided into four small regions. In the total area, we randomly scattered 100 nodes, the number of nodes in each region is: 21,29、23 and 27. The DFS is located in the middle of the total rectangular region. We assume that the nodes in the upper and the lower part of the optical region can communicate with each other, and the simulation results are shown in Fig 5.6.

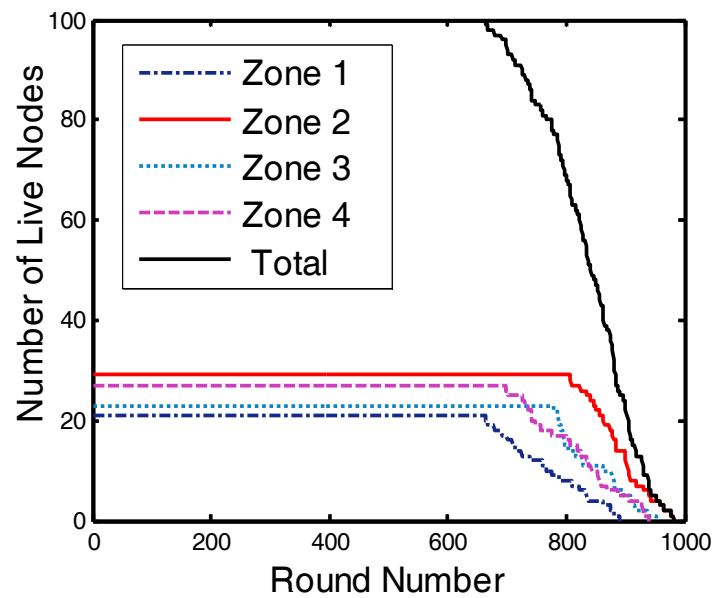

Fig. 5.6 Performance (\# of live nodes vs. round) after introducing the DFS into the rectangular sensor area

We can see from Fig. 5.6 that the numbers of the first dead node in the four regions are 663, 805,779 and 698, respectively. The first $20 \%$ nodes die slowly, but the remaining ones die 
rapidly in the total region. The results further demonstrate that the hybrid sensor network incorporating DFS with the O-LEACH protocol can evenly distribute the energy load among nodes, therefore prolong the overall lifetime of the network.

\section{Conclusion}

We discussed several improved algorithms (protocols) that can be used for WSNs or hybrid sensor networks with distributed fiber sensors involved. As sensor networks are much more complicated in real applications, more thorough and careful optimization of routing algorithms are required to meet specific requirements, such as real-time, long lifetime, security, and so on.

\section{References}

[1] I. F. Akyildiz, W. Su, Y. Sankarasubramaniam, E. Cayirci (2002). A survey on sensor networks, IEEE Communication Magazine, vol. 40, no.8, pp.102-114

[2] J. M. Kahn, R. H. Katz, and K. S. J. Pister (1999). Next century challenges: mobile networking for smart dust, Proc. ACM MobiCom '99, Washington DC, pp. 271-78

[3] V. Rodoplu and T. H. Meng (1999). Minimum energy mobile wireless networks, IEEE JSAC, vol. 17, no. 8, pp.1333-1344

[4] K. Sohrabi et al. (2000). Protocols for self-organization of a wireless sensor network, IEEE Pers. Commun., pp.16-27

[5] W. R. Heinzelman, A. Chandrakasan, and H. Balakrishnan (2000). Energy-efficient communication protocol for wireless microsensor networks, IEEE Proc. Hawaii Int'l. Conf. Sys. Sci., pp. 1-10

[6] X. Fan, Y. Song (2007). Improvement on LEACH protocol of wireless sensor network, IEEE SENSORCOMM, pp.260-264

[7] H. Jeong, C.-S. Nam, Y.-S. Jeong, D.-R. Shin (2008). A mobile agent based LEACH in wireless sensor network, Conf. on Advanced Comm. Technol. (ICACT), pp. 75-78

[8] Stephanie Lindsey and Cauligi S. Raghavendra (2002). PEGASIS: Power-Efficient Gathering in Sensor Information System, 2002 IEEE Aerospace Conference, vol. 3, pp.1125-1130

[9] X. Bao, D. J. Webb, and D. A. Jackson (1993). 32-km distributed temperature sensor using Brillouin loss in optical fiber, Opt. Lett., vol. 18, pp.1561-1563.

[10] D. Garus, T. Gogolla, K. Krebber, F. Schliep (1997). Brillouin optical-fiber frequencydomain analysis for distributed temperature and strain measurements, J. Lightwave Technol., vol.15, no.4, pp.654-662

[11] S.M. Maughan, H. H. Kee, T. P. Newson (2001). A calibrated 27-km distributed fiber temperature sensor based on microwave heterodyne detection of spontaneous Brillouin scattered power, IEEE Photon. Technol. Lett., vol. 13, no 5, pp. 511-513

[12] J. C. Juarez, E. W. Maier, K. N. Choi, H. F. Taylor (2005). Distributed fiber-optic intrusion sensor system, J. Lightwave Technol. vol.23, no.6, pp.2081-2087

[13] D. Iida, F. Ito (2008). Detection sensitivity of Brillouin scattering near Fresnel reflection in BOTDR measurement, J. Lightwave Technol., vol. 26, no.4, pp.417-424

[14] D. Kedar and S. Arnon (2003). Laser 'Firefly' Clustering; a New Concept in Atmospheric Probing, IEEE Photon. Tech. Lett., vol.15, no.1 pp. 1672-1624 
[15] S. Teramoto, and T. Ohtsuki (2004). Optical wireless sensor network system using corner cube retroreflectors (CCRs), IEEE Globecom'04, pp.1035-1039

[16] D. Kedar, S. Arnon (2005). Second generation laser firefly clusters: an improved scheme for distributed sensing in the atmosphere, Appl. Opt., vol. 44, no.6, pp.984992

[17] Jamal N. AL-Karaki, Ahmed E. Kamal (2004). Routing Techniques in Wireless Sensor Networks: A Survey, IEEE Wireless Communications, Dec.

[18] W. Heinzelman, J. Kulik, and H. Balakrishnan (1999). Adaptive Protocols for Information Dissemination in Wireless Sensor Networks, Proc. 5th ACM/IEEE Mobicom, Seattle, WA, pp. 174-85.

[19] J. Kulik, W. R. Heinzelman, and H. Balakrishnan (2002). Negotiation-Based Protocols for Disseminating Information in Wireless Sensor Networks, Wireless Networks, vol. 8, pp. $169-85$.

[20] Wendi Beth Heinzelman (2000). Application-Specific Protocol Architectures for Wireless Networks (PhD), Boston: Massachusetts Institute of Technology

[21] Vivek Mhatre, Catherine Rosenberg (2004). Design guidelines for wireless sensor networks: Communication, clustering and aggregation, Ad Hoc Networks, vol.2, no.1, pp. $45-63$

[22] Ning Xu, Sumit Rangwala, Krishna Kant Chintalapudi, Deepak Ganesan, Alan Broad, Ramesh Govindan, Deborah Estrin (2004). A Wireless sensor network for structural monitoring, Proc. 2nd international conference on Embedded networked sensor systems, Baltimore, MD, USA, pp.13-24.

[23] Katayoun Sohrabi, Jay Gao, Vishal Ailawadhi , Gregory J.Pottie (2000). Protocols for Self-organization of a Wireless Sensor Network, IEEE Personal Communications, vol.7, no.5, pp.16-27

[24] ISO.16484-5, Building automation and control systems part 5 data communication protocol, 2003.

[25] Stephanie Lindsey, Cauligi Raghavendra, Krishna M. Sivalingam (2002). Data Gathering Algorithms in Sensor Networks Using Energy Metrics, IEEE Transactions on Parallel and Distributed Systems, vol.13, no.9, pp.924-935 
www.intechopen.com 


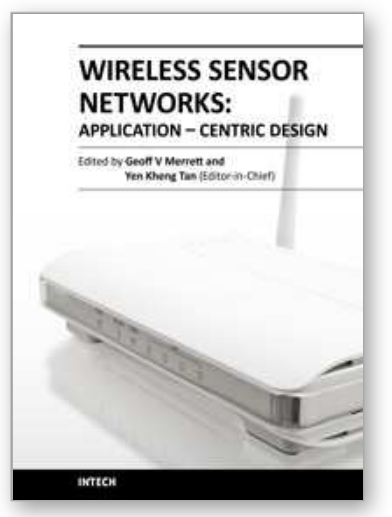

\author{
Wireless Sensor Networks: Application-Centric Design \\ Edited by Yen Kheng Tan
}

ISBN 978-953-307-321-7

Hard cover, 492 pages

Publisher InTech

Published online 14, December, 2010

Published in print edition December, 2010

Over the past decade, there has been a prolific increase in the research, development and commercialisation of Wireless Sensor Networks (WSNs) and their associated technologies. WSNs have found application in a vast range of different domains, scenarios and disciplines. These have included healthcare, defence and security, environmental monitoring and building/structural health monitoring. However, as a result of the broad array of pertinent applications, WSN researchers have also realised the application specificity of the domain; it is incredibly difficult, if not impossible, to find an application-independent solution to most WSN problems. Hence, research into WSNs dictates the adoption of an application-centric design process. This book is not intended to be a comprehensive review of all WSN applications and deployments to date. Instead, it is a collection of state-of-the-art research papers discussing current applications and deployment experiences, but also the communication and data processing technologies that are fundamental in further developing solutions to applications. Whilst a common foundation is retained through all chapters, this book contains a broad array of often differing interpretations, configurations and limitations of WSNs, and this highlights the diversity of this ever-changing research area. The chapters have been categorised into three distinct sections: applications and case studies, communication and networking, and information and data processing. The readership of this book is intended to be postgraduate/postdoctoral researchers and professional engineers, though some of the chapters may be of relevance to interested masterâ $€^{\mathrm{TM}} \mathrm{s}$ level students.

\title{
How to reference
}

In order to correctly reference this scholarly work, feel free to copy and paste the following:

Xiaoyin Li, Wei Pan, Jiangtao Liu, Zhen Zhang and Lianshan Yan (2010). Hybrid Optical and Wireless Sensor Networks, Wireless Sensor Networks: Application-Centric Design, Yen Kheng Tan (Ed.), ISBN: 978-953-307321-7, InTech, Available from: http://www.intechopen.com/books/wireless-sensor-networks-application-centricdesign/hybrid-optical-and-wireless-sensor-networks

\section{INTECH}

open science | open minds

\section{InTech Europe}

University Campus STeP Ri

Slavka Krautzeka 83/A

51000 Rijeka, Croatia

Phone: +385 (51) 770447

\section{InTech China}

Unit 405, Office Block, Hotel Equatorial Shanghai

No.65, Yan An Road (West), Shanghai, 200040, China

中国上海市延安西路65号上海国际贵都大饭店办公楼 405 单元

Phone: +86-21-62489820 
Fax: +385 (51) 686166

Fax: +86-21-62489821

www.intechopen.com 
(C) 2010 The Author(s). Licensee IntechOpen. This chapter is distributed under the terms of the Creative Commons Attribution-NonCommercialShareAlike-3.0 License, which permits use, distribution and reproduction for non-commercial purposes, provided the original is properly cited and derivative works building on this content are distributed under the same license. 